\title{
Omics-based identification of shared and gender disparity routes in Hras12V-induced hepatocarcinogenesis: an important role for Dlk1- Dio3 genomic imprinting region
}

Huiling Li

Dalian Medical University https://orcid.org/0000-0002-1724-2087

Jing Zhang

Dalian Medical University

Jianyi Dong

Dalian Medical University

Xu Zheng

Dalian Medical University

Chuanyi Lei

Dalian Medical University

Hong Wang

Dalian Medical University

Nan Zhang

Dalian Medical University

Yang Liu

Dalian Medical University

Xiaoqin Luo

Dalian Medical University

Jun Chen

Dalian Medical University

Jingyu Wang

Dalian Medical University

Aiguo Wang ( $1062880793 @ q q . c o m$ )

https://orcid.org/0000-0001-5022-3585

Research article

Keywords: miRNAomics, hepatocarcinogenesis, gender disparity, Dlk1-Dio3 genomic imprinting region

Posted Date: February 11th, 2020 
DOI: https://doi.org/10.21203/rs.2.23077/v1

License: (c) (1) This work is licensed under a Creative Commons Attribution 4.0 International License. Read Full License 


\section{Abstract}

Background: The phenomenon of gender disparity is very profound in hepatocellular carcinoma (HCC). Although previous research has revealed important roles of microRNA (miRNA) in HCC, there are no studies investigating the role of miRNAs in gender disparity observed hepatocarcinogenesis.

Results: In the present study, we investigated the global miRNAomics changes related to Ras -induced male-prevalent hepatocarcinogenesis in a Hras12V -transgenic mouse model ( Ras - Tg) by nextgeneration sequencing (NGS). We identified shared by also unique changes in miRNA expression profiles in gender-dependent hepatocarcinogenesis. 264 differentially expressed miRNAs ( DEMIRs) with q value $\leq 0.05$ and fold change $\geq 2$ were identified. A vertical comparison revealed that the lower numbers of DEMIRs in the hepatic tumor $(T)$ compared with the peri-tumor precancerous tissue $(P)$ of Ras $-\mathrm{Tg}$ and normal liver tissue of wild-type C57BL/6J mice (W) in males indicated that males are more susceptible to develop HCC. The expression pattern analysis revealed 43 common HCC-related miRNAs and 4 Ras positive-related miRNAs between males and females. By integrating the mRNA transcriptomic data and using 3-node FFL analysis, a group of significant components commonly contributing to HCC between sexes were filtered out. A horizontal comparison showed that the majority of DEMIRs are located in the DIk1-Dio3 genomic imprinting region (GIR) and that they are closely related to not only hepatic tumorigenesis but also to gender disparity in hepatocarcinogenesis. This is achieved by regulating multiple metabolic pathways, including retinol, bile acid, and steroid hormones.

Conclusions: In conclusion, the identification of shared and gender-dependent DEMIRs in hepatocarcinogenesis provides valuable insights into the mechanisms that contribute to male-biased Ras -induced hepatic carcinogenesis.

\section{Background}

Hepatocellular carcinoma (HCC) is an extremely heterogeneous cancer; treatment options are limited, and its prognosis remains extremely poor. A male predominance in $\mathrm{HCC}$ incidence has been reported, with male-to-female ratios ranging between 2:1 and 8:1 [1-3]. Consistently, animal studies also revealed that hepatocarcinogenesis is more common in male rodents, regardless of its etiology (spontaneous, or induced chemically, genetically, or through chronic viral infection) [4-6]. Therefore, humans and animals may share common mechanisms contributing to the gender disparity of hepatocarcinogenesis; these mechanisms remain, however, largely unknown.

MiRNAs are small non-coding RNA molecules, consisting of approximately 22 nucleotides. They are conserved among several organisms, including plants, animals, and viruses. It is now clear that miRNAs they are involved in post-transcriptional regulation of gene expression [7]. It is becoming increasingly evident that miRNAs are involved in cancer development and progression, by exerting anti-tumor or tumor-promoting roles [8]. The abnormal expression of miRNAs is involved in several biological processes, including proliferation, apoptosis, and metastasis, and could serve as therapeutic targets and 
prognostic biomarkers for HCC [9]. However, there are no studies on the gender disparity of miRNA expression profiles related to hepatocarcinogenesis.

RAS gene is commonly mutated in the vast majority of all human tumors; their frequency is the highest among all genes associated with human cancers [10]. Moreover, Ras is mutated in $70 \%$ of murine HCCs. Despite the fact that activating mutations in RAS occur in only $5 \%$ of human HCC, the aberrant activation of the RAS/MAPK signal transduction pathway due to other mutations is very common in human HCC [11-13], supporting the notion that RAS plays a key role in hepatocarcinogenesis [14]. We have previously generated a Hras $12 \mathrm{~V}$ transgenic mouse line using an Hras12V-encoding construct, which provides a hepatocyte-specific expression of the Ras oncogene, resulting in multicentric spontaneous hepatic tumorigenesis which is highly reproducible and shows male prevalence [14]. This hepatic tumor animal model has led to the elucidation of several mechanisms underlying hepatocarcinogenesis $[15,16]$.

In the present study, we employed the Hras12V transgenic mice and next-generation sequencing (NGS) to investigate the global miRNAomic profile related to Ras-induced hepatic tumorigenesis with gender disparity. Moreover, by vertical comparison within males and females and horizontal comparisons between sexes, we identified shared, unique, and systemic signatures in hepatocarcinogenesis. Especially, the importance of Dlk1-Dio3 genomic imprinting region (GIR) in hepatocaricogenesis was found. In addition, by integrating transcriptomics data and using 3-node FFL analysis, a group of significant components contributing to HCC among sexes were identified.

\section{Results}

\section{Next-generation sequencing (NGS) identified gender-dependent miRNA expression profiles in hepatocarcinogenesis}

To detect miRNA expression profiling, the normal liver tissue samples (W) of wild-type non-transgenic mice (non- $\mathrm{Tg}$ ) and hepatic tumors $(\mathrm{T})$ and matched adjacent precancerous tissue samples $(\mathrm{P})$ of Hras12V transgenic mice (Ras-Tg) from males and females were collected and named MW, MP, MT, FW, FP, and FT (M indicates male; F indicates female), respectively (Fig. S1). The miRNA expression profiles of these samples were assessed by NGS technology from isolated total RNAs. For every sample, an average of 27 million (ranged from 20-36 million) miRNA raw reads were obtained and the average clean ratio for the raw reads was $98.87 \%$ (ranged from 97.97-99.48\%) (Table S1). Saturation analysis showed that the miRNAs sequenced and mapped by trimmed reads were saturated when the sequencing depth approached 10 million (Fig. S2), indicating that the sequencing depth used in our study was sufficient to achieved high transcriptome coverage. Pearson's correlation and principal component analysis (PCA) indicated that the miRNA expression profiles of the six tissue groups vary significantly (Fig. 1A, B). The miRNAs read values were transformed to TPM (transcripts per million), and, by using the criteria of qvalue $\leq 0.05$ and fold-change $\geq 2$, a total of 191 DEMIRs in males and 204 DEMIRs in females (Fig. 1C; Table S2) were identified at least in one paired comparison among $W, P$, and $T$. The identified miRNA 
expression profiling data revealed shared, but also unique expression patterns in hepatocarcinogenesis between sexes.

To assess the reliability of the quantitative miRNAomics analysis obtained by NGS, six of the identified differentially expressed miRNAs in both sexes were randomly selected and further evaluated in different males and females by RT-qPCR (Fig. 2). The expression changes of these miRNAs were consistent with the NGS data, both in males and in females (Table S2).

\section{MiRNA expression profiling revealed different distribution of DEMIRs during hepatocarcinogenesis between sexes}

To investigate the changes in miRNA expression profiles during hepatocarcinogenesis in each sex, we determined the numbers of miRNAs that their expression changed significantly, by pairwise comparison among $\mathrm{W}, \mathrm{P}$, and $\mathrm{T}$ of males and females. Interestingly, the changes in miRNA expression profiles differed between males and females (Fig. 3A, B). For both sexes, the most prominent changes were observed when $T$ was compared with $W$ and $P$, indicating that miRNAs may play crucial roles in hepatocarcinogenesis. In $T$ versus $W(T / W)$ and $T$ versus $P(T / P)$, the numbers of up-regulated miRNAs were approximately 3-4-fold higher than that of down-regulated miRNAs, implying that HCC-related miRNAs play a main role in the down-regulation of protein levels. Profoundly higher numbers of miRNAs whose expression was altered in T was observed in females, indicating that the aberrant regulation of a higher number of miRNAs is needed for hepatic transformation in females, reflecting the lower incidence of HCC observed in females. On the other hand, the number of miRNAs whose expression was altered in $\mathrm{P}$ versus $\mathrm{W}(\mathrm{P} / \mathrm{W})$ was higher in males, indicating that males are more susceptible to Ras-induced carcinogenesis. The opposite changes in numbers of up- and down-regulated miRNAs in P/W suggests that different mechanisms are involved in oncogene stress in males and females.

\section{Shared and unique miRNAs expression patterns during hepatocarcinogenesis between sexes}

To identify shared and unique miRNAs involved in hepatic tumorigenesis in males and females, Venn analysis was firstly performed for T/P, T/W, and P/W in males and females (Fig. 3C, D; Table S2). And then, we classified the miRNAs into four categories (from (a) to (b)) describing particular variation trends of miRNAs during hepatic tumorigenesis. Further, shared and unique miRNAs were identified, depending on the categories (Table S3). To focus on the clear and definite variation tendency, we summarized at least two times significantly changed miRNAs in pairwise comparison among W, P, and T (Fig. 3E) (Fig. S3 summarized the expression patterns for all detected 264 DEMIRs). 
In total, 144 miRNAs were found to show at least two times significant changes in a pairwise comparison between W, P, and T (Fig. 3E). Among them, 104 miRNAs were found in males, while 119 were found in females. There were 79 miRNAs shared to both sexes, while 25 and 40 miRNAs were unique in males and females, respectively. Among the shared miRNAs, 68 miRNAs were negatively associated with liver tumorigenesis. Among the unique miRNAs, 19 and 29 miRNAs were positively correlated to liver carcinogenesis in males and females, respectively, and 6 and 11 miRNAs were negatively correlated to hepatic tumors in males and females, respectively (Fig. 3E ; Table S3).

Additionally, the four categories of miRNA expression patterns can be further classified into several subtypes (Fig. 3E). The symbols used here indicate that ">" and "<": miRNAs were significantly up- and down-regulated, respectively; and "=": no significant difference in miRNA expression levels was observed between the samples. The category HCC-positive-related miRNAs (a) includes three subtypes: (1) $T>P=W$; (2) $T=W>P$; (3) $T>W>P$. The most miRNAs in this category were classified into subtype (1). The category Ras-positive-related miRNAs (b) includes two subtypes: (1) $\mathrm{W}<\mathrm{P}=\mathrm{T}$ and (2) $\mathrm{W}<\mathrm{P}<\mathrm{T}$. These miRNAs were equally (or gradually) and significantly up-regulated in $\mathrm{P}$ and $\mathrm{T}$ compared with $\mathrm{W}$. The category HCCnegative-related miRNAs (c) includes two subtypes: (1) $T<P=W,(2) T=W<P$. The most miRNAs in this category were classified into subtype (1). The category Ras-negative-related miRNAs (d) includes two types: (1) $W>T=P$ and (2) $W>P>T$. These miRNAs were equally (or gradually) and significantly downregulated in $\mathrm{P}$ and $\mathrm{T}$ compared with $\mathrm{W}$.

In particular, 38 miRNAs in (a)-(1) type and 5 miRNAs in (c)-(1) type occupy the most proportions of miRNAs in variant trend types, which represent the shared miRNAs involved in hepatic tumor development (Table S3). Additionally, 4 (in b) common miRNAs in both sexes were found to be positively related to Ras oncogene expression. Moreover, the higher number of unique miRNAs in females compared to males (40 vs. 25) suggests that more changes in miRNAs expression are required in females to develop HCC (Table S3).

\section{Identification of the regulatory networks that are involved in hepatocarcinogenesis both in males and females}

To identify the regulatory networks that are involved in hepatocarcinogenesis both in males and females, the common HCC-related miRNAs (38 up-regulated ( $T>P=W) ; 5$ down-regulated $(T<P=W)$ in Fig. $3 E$ ) and mRNAs (646 up-regulated ( $T>P=W) ; 323$ down-regulated $(T<P=W$ ) in Table $S 4)$ in males and females were selected for further analysis (Fig. 4I). Considering that mRNAs are negatively regulated by miRNAs, 323 down-expressed mRNAs may be regulated by 38 up-expressed miRNAs and 646 up-expressed mRNAs may results from 5 down-expressed miRNAs. Further, 35 TFs (27 in up-expressed and 8 in down- 
expressed mRNAs) were identified. Finally, four types of regulatory relationships among miRNAs, TFs, and genes were predicted (Fig. 4ll; Table S5; Table S6).

To merge the four types of regulatory relationships predicted above (Fig. 4II), 3-node FFLs were formed (Fig. 4III; Table S7; Table S8). The network contained 1182 edges and 326 unique nodes in the $920 \mathrm{FFLs.}$ Among the 1182 edges, 461 miRNA-gene, 4 miRNA-TF, 588 TF-gene, and 129 TF-miRNA pairs were predicted. Among the 326 nodes, 265 genes, 46 miRNAs, and 15 TFs were specific for HCC. Further, these FFLs were categorized into TF-FFLs (TF), miRNA-FFLs (miRNA), and composite-FFLs (composite) subnetworks (SNW) (Fig. 4IV). In these three SNWs, TF-SNW is the most redundant. By using the hub definition method proposed by Yu et al.[17], we determined the top 25\% degree nodes of gene, miRNA and

TF as the member of secondary TF-SNW to focus on the most significant components. To further analyze the potential function, all nodes in the three SNWs (secondary TF-SNW, miRNA-SNW, composite-SNW) were filtered based on the GEPIA and PUBMED database, identifying a group of significant components (Fig. 4V).

\section{Differentially expressed miRNAs (DEMIRs) between males and females}

To identify the DEMIRs between males and females involved in hepatic tumorigenesis, comparisons between the sexes (MW versus FW (MW/FW); MP versus FP (MP/FP); MT versus FT (MT/FT)) were performed. Interestingly, the highest number of DEMIRs was detected in MT/FT, and the lowest number of DEMIRs was detected in MP/FP (74 vs. 19), whereas 48 DEMIRs were identified in MW/FW (Fig. 5A). Venn diagram showed that the number of overlapped DEMIRs between MT/FT and MW/FW was higher than that between MP/FP and MT/FT (MW/FW) (Fig. 5B). Our findings suggest that RAS/ERK pathway activation reduces the differences in miRNA expression profiles between precancerous hepatocytes of the males and females. However, during hepatocarcinogenesis, the differences in miRNA expression profiles between hepatomas of the males and females are enhanced. The 29 overlapping miRNAs between MT/FT and MW/FW are possibly linked to the gender disparity of hepatocytes and hepatomas. Intriguingly, the opposite changes in numbers of up- and down-regulated miRNAs found between MW/FW and MT/FT suggest profound gender disparity of hepatocytes and hepatomas.

\section{The miRNA clusters in the DIk1-Dio3 genomic imprinting region (GIR) play important roles in hepatocarcinogenesis}

DIk1-Dio3 GIR is highly conserved in humans and mice, located on chromosome 14q32 and 12qF1, respectively. It contains three protein-coding genes and several non-coding RNA clusters (Fig. 5C). The miRNAs encoded in the GIR constitute the largest miRNAs cluster in both human and mouse and play crucial roles in most cancers, through the regulation of multiple pathways $[18,19]$. In the present study, 
$53.5 \%$ of the detected DEMIRs (54 out of 101) between the two sexes were found to be located on DIk1Dio3 GIR (Fig. 5D; Table S9). The variation of DEMIRs in DIk1-Dio3 GIR is consistent with that of total DEMIRs between sexes (Fig. 5A, D), suggesting that the DEMIRs in DIk1-Dio3 GIR may play crucial roles in gender-disparity of hepatocytes and hepatocarcinogenesis.

Moreover, $54.2 \%$ (26 out of 48) DEMIRs in MW/FW were located on DIk1-Dio3 GIR, and 25 of them were up-regulated in MW (Fig. 5D, E; Table S9). Intriguingly, in MP/FP, only 5.3\% (1 out of 19) DEMIR, miR-341$3 p$, is located in DIk1-Dio3 GIR (Fig. 5E; Table S9). This reflects the reduced gender disparity in miRNA expression in DIk1-Dio3 GIR under the expression of Ras oncogene. However, in MT/FT, 71.6\% (53 out of 74) DEMIRs were located in Dlk1-Dio3 GIR (Fig. 5E; Table S9), and in contrast to MW/FW, 53 miRNAs were down-regulated in MT. During tumorigenesis, the expression of miRNAs located in DIk1-Dio3 GIR decreased in $\mathrm{P}$ and then increase again in T compared to W in males (Fig. 5F; Table S9). However, in females, the expression of miRNAs in DIk1-Dio3 GIR tended to increase gradually in P and T compared to W (Fig. 5G; Table S9). These findings indicate that the overexpression of miRNAs of the Dlk1-Dio3 GIR plays a crucial role in hepatocarcinogenesis in both sexes. The significantly higher levels of these miRNAs in hepatoma in females compared to males imply their important role in the lower susceptibility of hepatocarcinogenesis observed in females.

Although the different expression patterns of miRNAs exist between males and females, the vast majority of DEMIRs located in DIk1-Dio3 GIR were up-regulated in T comparing to $P$ in both sexes (Fig. 5F, G). This finding indicates that the up-regulation of miRNAs located in DIk1-Dio3 GIR may have similar and important roles in hepatocarcinogenesis in both sexes. In order to understand the functions of these DEMIRs on DIk1-Dio3 GIR in hepatic tumorigenesis, their target genes were predicted using the TargetScan and miRDB database among the common 530 down-regulated mRNAs in T/P from both sexes (Table S10). Target enrichment analysis was performed using the Metascape database. Key metabolic pathways were identified. Among them, pathways related to retinol, steroid, lipid, bile acid, and bile salt were highly enriched (Table 1 ; Table S10). These results indicate that the miRNA encoded by DIk1-Dio3 GIR play important roles in hepatocarcinogenesis by regulating multiple metabolic pathways.

\section{Discussion}

Even though microRNAs (miRNAs) do not encode for any proteins, they play very important roles in the regulation of gene expression at the post-transcriptional level. The miRNA expression profiles observed in HCC have been extensively studied in the recent years. Multiple profiling studies have revealed that miRNA expression profiles are substantially different between human HCC and healthy livers [20, 21]. However, the gender disparity of microRNAomics related to hepatic tumorigenesis is largely understudied. 
Clinically, due to the limitations of collecting matched specimens from sex-based HCC patients, such as complex etiologies and subclasses, different development stages, difficulties of obtaining normal liver tissues, etc., mechanisms underlying gender-dependent hepatocarcinogenesis are rarely investigated. Therefore, animal models provide valuable opportunities to shed light on clinical investigations for dissecting the underlying mechanisms responsible for this gender disparity. Especially, the matched wild type normal liver tissue and precancerous liver tissue for hepatic tumors provide opportunity for further dynamic change processes analysis during hepatocarcinogenesis. In the present study, we classified the miRNA expression patterns into four categories and several subtypes (Fig. 3E), and the following meaningful informations are obtained: (1) the DEMIRs classified into (a)-(1) $(T>P=W)$ and (c)-(1) $(T<P$ $=\mathrm{W})$ subtypes indicate that these miRNAs are total absence or weak changes in response to Ras expression in precancerous hepatocytes and play predominantly positive and negative roles in hepatic tumors, respectively; (2) the DEMIRs classified into (a)-( 2$)(T=W>P)$ and (a)-(3) $(T>W>P)$ subtypes indicate that these miRNAs have a reverse relationship with oncogene expression in precancerous hepatocytes and the expression of these miRNAs is potentially regulated as a part of a tumor defense system; (3) the DEMIRs classified into (c)-(2) $(T=W<P)$ subtype indicates that these miRNAs are positively correlated to Ras expression in precancerous hepatocytes and are potentially regulated by the tumor defense system; (4) the DEMIRs classified into (b) and (d) categories indicates that these miRNAs are positive and negative correlation to the Ras oncogene in both precancerous hepatocytes and hepatoma cells, respectively. Above novel findings provided valuable clues and insights for further researches.

The miRNAomics data obtained in the present study suggested that, even though the alterations in the miRNA expression profiles during Ras-induced hepatic tumorigenesis occurred in a different manner, common changes between males and females were observed as well. Intriguingly, the most profound changes were observed in $T$ when compared to $P$ and $W$ (Fig. 3A, B). This was consistent in males and females, and it highlights that miRNAs may play crucial roles in hepatocarcinogenesis. Among all 264 detected DEMIRs, 43 miRNAs (38 up-regulated and 5 down-regulated in T) were found to be related to HCC and were shared between males and females (Fig. 3E). Integrating analysis with the RNA sequencing data, the 3-node FFLs were formed and finally filtered out a group of significant components (Fig. 5V). Among them, miR-376c-3p, miR-664, miR-484, miR-23a, miR-137, RUNX2, and Ndrg2 had been reported to be closely involved in hepatic tumorigenesis and development [22-29]. It indicates the reliability of the 3-node FFLs analysis. Although the remained genes filtered out in the present study such as Akr1d, Apoa5, Crp, Cxcl12, Kcnk5, Ngfr, Rnf152, Tmem25, Ptgfrn, and Slc6a8 are understudied, the same expression patterns of these genes in mouse and human HCC indicate their important roles in hepatocarcinogenesis and provide a primary reference and potential targets for further investigations.

Deregulation of miRNAs encoded by the DIk1-Dio3 GIR has been associated with various malignancies, including HCC $[18,19]$. A clinical study reported that the overexpression miRNA found in this cluster was strongly associated with the expression of several HCC stem cell markers, including CD133, CD90, EpCAM, and Nestin. Moreover, high expression levels of these miRNAs correlated with a high level of serum Alpha Fetoprotein (AFP) and worse prognosis in HCC patients [30]. Additionally, previous studies 
have demonstrated that miRNAs encoded by the DIk1-Dio3 GIR, including miR-127, miR-411, miR-370, miR-134, and miR-1188, are closely associated with the hepatic tumors [31-35]. In agreement with that, most of the DEMIRs identified in the present study were also found to be located encoded by Dlk1-Dio3 GIR (Fig. 5). Especially, in our experimental system, we revealed that the miRNA clusters located on DIk1Dio3 GIR are closely related to not only hepatic tumorigenesis but also the gender disparity observed in HCC. This new evidence provides an insight in the importance of Dlk1-Dio3 GIR in gender-dependent hepatocarcinogenesis.

The target pathway enrichment analysis revealed that DEMIRs encoded by DIk1-Dio3 GIR might have a crucial role in driving hepatocarcinogenesis by regulating multiple tumor-associated metabolic pathways (Table 1). Importantly, interference with retinol metabolism may promote hepatocarcinogensis. Retinol has been reported to exert cytostatic effects in human HCC cells, as well as to promote programmed cell death and cell cycle arrest at the G0/G1 phase [36]. Acyclic retinoid (ACR) has also been reported to have chemopreventive effects in rodent HCC models [37, 38]. In line with this, clinical studies have shown that ACR can lead to a significantly lower incident of post-therapeutic recurrence in HCC patients, and as well as to improved outcomes $[39,40]$. Additionally, the down-regulation of the synthesis of the water-soluble steroids bile acids (BAs) and bile salts may result in the accumulation of cholesterol, which further facilitates the hepatic tumorigenesis and HCC progression. Disruption in BA level balance has been linked to several pathological conditions, including tumorigenesis, suggesting BAs to have potential tumorpromoting roles [41]. BAs have also been shown to act as strong cues that lead to DNA damage and DNAdamage-induced apoptosis; sustained exposure could, therefore, lead to cells that are less susceptible to apoptosis [42]. Moreover, the steroid metabolic process was significantly inhibited. Several steroids, including cholesterol and sex hormones, have been considered to be tumor-related factors. More specifically, cholesterol levels are often higher in HCC cells compared to normal liver cells [43], and the relatively lower serum level of cholesterol observed in HCC patients may be due to cirrhosis, which is observed in $80 \%$ of HCC patients [44]. Several clinical studies have also provided evidence that cholesterol has a potential contribution to liver malignancies $[45,46]$. Androgens are also believed to exert tumor-promoting roles in HCC, while estrogen is believed to have a protective role. Therefore, sex hormones not only play crucial roles in regulating the differences in sex characteristics and biological processes between males and females but are also believed to contribute to gender-dependent hepatocarcinogenesis [47]. In summary, the target pathway enrichment analysis shows that DEMIRs on DIk1-Dio3 GIR may affect hepatocarcinogenesis by regulating multiple tumor-associated metabolic pathways.

The Hras12V-transgenic hepatic cancer mouse model has been previously described in detail [14]. Briefly, Hras12V-transgenic mice express the liver-specific Hras12V oncogene, resulting in liver cancer development at the definite stage and with considerable gender disparity. Namely, compared to Ras-Tg males, the hepatic tumorigenesis in females is characterized by late-onset, low incidence, slow progression, and small tumor size. In detail, male Ras-Tg mice develop HCC relatively stage, usually between 8 and 9 months of age); the incidence of HCC in these mice is extremely high (closed to 100\%). HCC tends to progress rapidly, and the mice eventually die of hepatic tumors at 12-14 months of age. In 
contrast, female Ras-Tg mice develop hepatic tumors at a much lower rate $(\sim 30 \%)$, and this happens at a much later stage (15-month-old) compared to male mice. Therefore, although age-related factors may be involved, to investigate the miRNAomics involved in the Ras-driven gender-dependent HCC, male and female mice aged 9- and 15-months were must be used, respectively. Our investigation had indicated that differences in molecular responses to deregulated RAS oncoprotein between Ras-Tg females and males determine the onset of HCC development [14]. Further, by exploring the advanced omics-based molecular techniques, our previously published global proteomics and metabonomics data had revealed genderdependent routes for hepatocarnigenesis $[15,16]$. In the present study, the gender disparities were also reflected in the miRNAomics data. As the humans and rodents were supposed to share the common routes for gender dependent HCC, the Ras-Tg combined with its related global and systemic omics-data will provide a valuable model for elucidating the underlying mechanisms contributing to the gender disparity of hepatocarcinogenesis.

\section{Conclusions}

In conclusion, to our best knowledge, this is the first study to present global microRNAomics data related to gender disparity in Ras-induced HCC. The shared and gender-dependent biological and molecular changes that occur during hepatic tumorigenesis offer valuable clues in elucidating the mechanisms that contribute to male-biased Ras-induced hepatocarcinogenesis. The differentially expressed miRNAs identified in the hepatic tumors will add important biological information to tumor-related bioinformatics databases. Particularly, the overexpression of miRNA clusters on DIk1-Dio3 GIR was identified as a promoting factor in hepatocarcinogenesis by regulating multiple key cellular pathways.

\section{Methods}

\section{Animals handing and tissue sampling}

Procedures involving animal maintenance and handling, as well as tissue sampling were approved by the Animal Care and Use Committee of Dalian Medical University. Non-transgenic C57BL/6J mice (Non-Tg, purchased from Institute of Genome Engineered Animal Models for Human Disease in Dalian Medical University) and Hras12V-transgenic mice (Ras-Tg, a kind gift from Dr. Dae-Yeul Yu at Korea Research Institute of Bioscience and Biotechnology) were used for this study. Both strains were bred and housed in pathogen-free animal facility of the Laboratory Animal Center of Dalian Medical University. All animals were fed a standard normal diet (Liaoning Changsheng Biotechnology Co., Ltd.) ad libitum with free access to water and housed under controlled temperature (around $23^{\circ} \mathrm{C}$ ) and relative humidity $(40 \%-60 \%)$ conditions with a 12-12 hour light-dark cycle. To assess the male bias of hepatic tumorigenesis of RasTg, 9-month-old male and 15-month-old female Ras-Tg bearing hepatic tumors of the same stage were harvested. Healthy liver tissues from non-Tg males and females were also sampled as control. After the mice were culled and subjected to euthanasia by cervical dislocation, normal liver tissue samples from male and female non- $\mathrm{Tg}(\mathrm{W})$ and hepatic tumors $(\mathrm{T})$ and matched adjacent precancerous tissue samples 
(P) of Ras-Tg were collected. Tissue samples were flash-frozen in liquid nitrogen. Tissue samples were also fixed in $10 \%$ formalin and subsequently used for histopathological examination.

\section{Experimental design}

The Ras-Tg used had hepatic tumors at the same stage. Seven representative individuals were selected from each group, and small RNA samples were prepared individually. Mixing samples of experimental animal tissues of the same group is one of the most frequently used methods of quantitative sequencing, especially for inbred strains, which are nearly identical to each other in terms of genomic background. In addition, in the case of Ras-Tg, the hepatic tumors were protruded from the peri-tumor tissues and had a clear boundary between them, allowing for precise sampling yet a small chance to mix the hepatic tumor tissues with the peri-tumor tissues does exist. Therefore, seven individual samples of the same group were equivalently mixed to generate one combined sample. This design ensures sample coverage and reliable NGS results, which reflect the shared changes during hepatic tumorigenesis with a male bias. NGS approaches were performed to quantify the miRNA omics for MW, MP, MT, FW, FP, and FT. The workflow is depicted in Fig. S1.

\section{Quantitative microRNA-omics analysis by NGS}

MiRNA expression profiles were assessed using NGS analysis. miRNAs were isolated from W, P, and T tissues (7 individuals for each group); The RNA extraction was conducted using the mirVana ${ }^{\text {TM }}$ miRNA Isolation Kit (AM1561, Austin TX, US) according to the manufacturer's protocols. The total RNA quality and quantity were verified by electrophoresis using an Agilent 2100 Bioanalyzer (Agilent Technologies Santa Clara, US). Due to the fact that the genetic background of the mice used, the common hepatocarcinogenesis etiology (Ras-driven), and the high similarity in the pathological characteristics of the samples from each group, the RNA samples from the same group were mixed at equivalent proportions to generate six composite samples, i.e., W of male and female non-Tg (MW and FW, respectively), $\mathrm{P}$ of male and female Ras-Tg (MP and $\mathrm{FP}$, respectively), and $\mathrm{T}$ of male and female $\mathrm{Ras}-\mathrm{Tg}$ (MT and FT, respectively), to find out common variations against interindividual differences in the same group. MiRNA libraries were constructed with the TruSeq Small RNA Sample Prep Kit (Illumina, San Diego, CA, USA). The Illumina HiSeq 2000 (Illumina, San Diego, CA, USA) instrument was employed to sequence the miRNA library. Contaminating and low-quality reads were removed using the Fastx software (version: 0.0.13, http://hannonlab.cshl.edu/fastx_toolkit/index.html).

\section{Identification of differentially expressed miRNAs}


The RNA-seq data were used to identify the differentially expressed miRNAs (DEMIRs). Firstly, clean reads of 18-44 nt were mapped onto the reference genome (GRCm38, ftp:// ftp.ensembl.org/pub/release83/fasta/mus_musculus/dna/Mus_musculus.GRCm38.dna.primary_assembly.fa) using Bowtie [48] MiRNAs and other small RNAs were identified according to the location information of known miRNAs in miRBase, as described previously [49], and the location information of other small RNAs in the reference genome. In order to make the miRNA expression levels between different miRNAs and between different samples comparable, the reads of each miRNA were normalized using the TMM (trimmed mean of M values) method [50] and then converted into TPM (Transcripts per million; the formula used is: reads number on a miRNA $\times 10^{6} /$ total reads number) for the standardization [51]. Fold-change was calculated according to TPM, and Principal Component Analysis (PCA) was performed. EdgeR [52] was used to identify DEMIRs in paired comparisons between $\mathrm{W}, \mathrm{P}$, and $\mathrm{T}$, using the mapped reads. Filtering was performed using q-value $\leq 0.05$ and fold-change $\geq 2$ as threshold.

\section{Validation of DEMIRs by RT-qPCR}

To confirm the validity and reproducibility of our miRNAomics analysis findings, six of the identified differentially expressed miRNAs in both sexes were randomly selected and further evaluated by RT-qPCR analysis in samples from another set of males and females. The cDNA was synthesized using the Mir-X miRNA First-Strand Synthesis Kit (TaKaRa, Dalian, China) in accordance with the manufacturer's instructions. Quantitative real-time PCR (RT-qPCR) reactions were conducted with a StepOnePlus ${ }^{\mathrm{TM}}$ RealTime PCR System (ThermoFisher Scientific, CA, USA) using SYBR Green qPCR Master Mix (TaKaRa, Dalian, China). Mouse small nucleolar RNA, C/D box 68 (Snord68) was used as a reference gene for normalization [53]. The amount of miRNA relative to the internal control Snord 68 was calculated using

the $\Delta \Delta \mathrm{Ct}$ method (2-(Ct miRNA - Ct Snord68) $)$. The primers for the miRNAs were synthesized by Dalian Saituo biotechnology company (Dalian, China). The primer sequences are shown in Table S11.

\section{Identification of common regulatory networks related to hepatocarcinogenesis}

Differential mRNA expression analysis by NGS has been reported in our previously published study[31]. Considering that mRNAs are negatively regulated by miRNAs, the 3-node FFL co-regulatory network was built by exploring miRNA and mRNA-omics. Firstly, the miRNA-gene pairs were extracted using the TargetScan (release 7.1) [54] and miRDB [55] database. The miRNA-mRNA pairs with a total context score $<-0.3$ in TargetScan and target score $>50$ in miRDB were extracted. The two sets of miRNA-gene pairs were merged, and the mRNAs present in both databases were considered to be miRNA targets. Transcription factors (TFs) were retrieved from differently expressed mRNAs using the TRANSFAC Professional Database (release 2018.2) [56]. To assess the regulation of miRNA and mRNAs by TFs, the miRNA promoter region sequences (-2000 around TSS) and the promoter region sequences $(-2000 /+500$ around TSS) of genes involved in HCC were obtained from the UCSC database. These sequences were 
subsequently investigated for TF-binding sites, using the TRANSFAC Professional Database. Precalculated cut-offs were used to eliminate false-positive (minFP) matches and construct a high-quality matrix. We also limited the search to a core score of 1.00 , matrix score $>0.95$, and TFs that only belong to the mouse genome. Finally, four types of regulatory relationships among miRNAs, TFs, and genes were predicted.

The 3-node feed-forward loop (3-node FFL) is extremely common among transcriptional networks, and it can be broadly divided into three categories based on their inside regulations: miRNA-FFL, TF-FFL, and composite-FFL. In TF-FFL, the TF regulates the miRNA and the gene, while the miRNA represses the target gene. In miRNA-FFL, the miRNA represses both TF and gene expression while the TF regulates target gene expression. In composite-FFL, the TF regulates the miRNA and target gene while the miRNA represses the TF and the gene. The three types of FFLs are exclusive to each other. Therefore, three types of FFLs (miRNA-FFL, TF-FFL, and composite FFL) were extracted from the four categories of regulation relationships, and then the sub-networks (SNWs) were visualized using Cytoscape (version 3.6.1).

\section{Identification of significant components}

In the three SNWs, TF-SNW is the most redundant. To focus on the most significant components, the top $25 \%$ degree nodes of gene, miRNA and TF were determined as the member of secondary TF-SNW by using the hub definition method [17]. All nodes in the three SNWs (secondary TF-SNW, miRNA-SNW, composite-SNW) were filtered based on the GEPIA [57] and PUBMED database. The nodes have the same expression trend in human GEPIA database or their roles in hepatocarcinogenesis elucidated were considered to be significant components.

\section{Pathway enrichment analysis}

The target pathway enrichment analysis was performed by Metascape database [58].

\section{Abbreviations}

DEMIRs, differentially expressed miRNAs; F, females; FW, normal liver tissues of non-transgenic 15 months old female mice; FP, normal peri-tumor precancerous tissues of transgenic 15 months old female mice; FT, hepatic tumor tissues of transgenic 15 months old female mice; GIR, genomic imprinting region; HCC, hepatocellular carcinoma; M, male; miRNA, microRNA; MP, normal peri-tumor precancerous tissues of transgenic 9 months old male mice; MW, normal liver tissues of non-transgenic 9 months old male 
mice; MT, hepatic tumor tissues of transgenic 9 months old male mice; NGS, next generation sequencing; non-Tg, C57BL/6J wild type non-transgenic mice; P, peri-tumor precancerous tissue of Ras-Tg; PCA, principal component analysis; P/W, P versus W; 3-node FFL, 3-node feed-forward loop; SNWs, subnetworks; T, hepatic tumor of Ras-Tg; TPM, transcripts per million; T/P, T versus $\mathrm{P} ; \mathrm{T} / \mathrm{W}, \mathrm{T}$ versus W; Ras$\mathrm{Tg}$, Hras12V transgenic mice; W, normal liver tissues of non-Tg.

\section{Declarations}

\section{Ethics approval and consent to participate}

Experiments and procedures for animal handling and tissue sampling were approved by the Animal Care and Use Committee of Dalian Medical University.

\section{Funding}

This work was supported by the National Natural Science Foundation of China (30872950). This funding body had no role in the design of the study, collection, analysis, and interpretation of data, or in writing the manuscript.

\section{Authors' contributions}

A.W. and J.W. designed experiments, provided research funds and corrected the manuscript; J.Z., H.L., J.D., X.Z., C.L., H.W., N.Z., Y.L., X,L., J.C, and A.W. performed experiments and analyzed the data; J.Z., H.L., and A.W. co-wrote the manuscript. All authors read and approved the final manuscript.

\section{Consent for publication}

All contributing authors agree to the publication of this article.

Competing InterestThe authors declared that they have no conflicts of interest to this work.

\section{Availability of data and materials}


Not Applicable

\section{Acknowledgements}

Not Applicable

\section{References}

1. Wang AG, Moon HB, Chun SY, Lee TH, Yu DY, Lee DS: Orchiectomy reduces hepatotumorigenesis of H-ras12V transgenic mice via the MAPK pathway. Life Sci 2006, 79(21):1974-1980.

2. Hartwell HJ, Petrosky KY, Fox JG, Horseman ND, Rogers AB: Prolactin prevents hepatocellular carcinoma by restricting innate immune activation of $\mathrm{c}-\mathrm{Myc}$ in mice. Proceedings of the National Academy of Sciences of the United States of America 2014, 111(31):11455-11460.

3. El-Serag HB: Epidemiology of viral hepatitis and hepatocellular carcinoma. Gastroenterology 2012, 142(6):1264-1273.e1261.

4. Nakatani T, Roy G, Fujimoto N, Asahara T, Ito A: Sex hormone dependency of diethylnitrosamineinduced liver tumors in mice and chemoprevention by leuprorelin. Japanese journal of cancer research : Gann 2001, 92(3):249-256.

5. Naugler WE, Sakurai T, Kim S, Maeda S, Kim K, Elsharkawy AM, Karin M: Gender disparity in liver cancer due to sex differences in MyD88-dependent IL-6 production. Science (New York, NY) 2007, 317(5834):121-124.

6. Brandon-Warner E, Walling TL, Schrum LW, McKillop IH: Chronic ethanol feeding accelerates hepatocellular carcinoma progression in a sex-dependent manner in a mouse model of hepatocarcinogenesis. Alcoholism, clinical and experimental research 2012, 36(4):641-653.

7. Bartel DP: MicroRNAs: genomics, biogenesis, mechanism, and function. Cel/ 2004, 116(2):281-297.

8. Ji W, Sun B, Su C: Targeting MicroRNAs in Cancer Gene Therapy. Genes 2017, 8(1).

9. Maluccio $\mathrm{M}$, Covey $\mathrm{A}$ : Recent progress in understanding, diagnosing, and treating hepatocellular carcinoma. CA: a cancer journal for clinicians 2012, 62(6):394-399.

10. Hunter T: Oncoprotein networks. Cel/ 1997, 88(3):333-346.

11. Newell P, Toffanin S, Villanueva A, Chiang DY, Minguez B, Cabellos L, Savic R, Hoshida Y, Lim KH, Melgar-Lesmes $\mathrm{P}$ et al: Ras pathway activation in hepatocellular carcinoma and anti-tumoral effect of combined sorafenib and rapamycin in vivo. Journal of hepatology 2009, 51(4):725-733.

12. Taketomi A, Shirabe K, Muto J, Yoshiya S, Motomura T, Mano Y, Ikegami T, Yoshizumi T, Sugio K, Maehara Y: A rare point mutation in the Ras oncogene in hepatocellular carcinoma. Surgery today 2013, 43(3):289-292.

13. Delire B, Starkel P: The Ras/MAPK pathway and hepatocarcinoma: pathogenesis and therapeutic implications. European journal of clinical investigation 2015, 45(6):609-623. 
14. Wang AG, Moon HB, Lee MR, Hwang CY, Kwon KS, Yu SL, Kim YS, Kim M, Kim JM, Kim SK et al: Gender-dependent hepatic alterations in H-ras12V transgenic mice. Journal of hepatology 2005, 43(5):836-844.

15. Rong Z, Fan T, Li H, Li J, Wang K, Wang X, Dong J, Chen J, Wang F, Wang J et al: Differential Proteomic Analysis of Gender-dependent Hepatic Tumorigenesis in Hras12V Transgenic Mice. Mol Cell Proteomics 2017, 16(8):1475-1490.

16. Fan T, Rong Z, Dong J, Li J, Wang K, Wang X, Li H, Chen J, Wang F, Wang J et al: Metabolomic and transcriptomic profiling of hepatocellular carcinomas in Hras $12 \mathrm{~V}$ transgenic mice. Cancer medicine 2017, 6(10):2370-2384.

17. Yu H, Greenbaum D, Xin Lu H, Zhu X, Gerstein M: Genomic analysis of essentiality within protein networks. Trends in genetics : TIG 2004, 20(6):227-231.

18. Enfield KS, Martinez VD, Marshall EA, Stewart GL, Kung SH, Enterina JR, Lam WL: Deregulation of small non-coding RNAs at the DLK1-DIO3 imprinted locus predicts lung cancer patient outcome. Oncotarget 2016, 7(49):80957-80966.

19. Enterina JR, Enfield KSS, Anderson C, Marshall EA, Ng KW, Lam WL: DLK1-DIO3 imprinted locus deregulation in development, respiratory disease, and cancer. Expert review of respiratory medicine 2017, 11(9):749-761.

20. Murakami Y, Yasuda T, Saigo K, Urashima T, Toyoda H, Okanoue T, Shimotohno K: Comprehensive analysis of microRNA expression patterns in hepatocellular carcinoma and non-tumorous tissues. Oncogene 2006, 25(17):2537-2545.

21. Jiang J, Gusev Y, Aderca I, Mettler TA, Nagorney DM, Brackett DJ, Roberts LR, Schmittgen TD: Association of MicroRNA expression in hepatocellular carcinomas with hepatitis infection, cirrhosis, and patient survival. Clinical cancer research : an official journal of the American Association for Cancer Research 2008, 14(2):419-427.

22. Wang Y, Chang W, Chang W, Chang X, Zhai S, Pan G, Dang S: MicroRNA-376c-3p Facilitates Human Hepatocellular Carcinoma Progression via Repressing AT-Rich Interaction Domain 2. Journal of Cancer 2018, 9(22):4187-4196.

23. He JH, Han ZP, Liu JM, Zhou JB, Zou MX, Lv YB, Li YG, Cao MR: Overexpression of Long Non-Coding RNA MEG3 Inhibits Proliferation of Hepatocellular Carcinoma Huh7 Cells via Negative Modulation of miRNA-664. Journal of cellular biochemistry 2017, 118(11):3713-3721.

24. Qiu L, Huang Y, Li Z, Dong X, Chen G, Xu H, Zeng Y, Cai Z, Liu X, Liu J: Circular RNA profiling identifies circADAMTS13 as a miR-484 sponge which suppresses cell proliferation in hepatocellular carcinoma. Molecular oncology 2018.

25. Huang H, Liu Y, Yu P, Qu J, Guo Y, Li W, Wang S, Zhang J: MiR-23a transcriptional activated by Runx2 increases metastatic potential of mouse hepatoma cell via directly targeting Mgat3. Scientific reports 2018, 8(1):7366.

26. Cao Z, Sun B, Zhao X, Zhang Y, Gu Q, Liang X, Dong X, Zhao N: The Expression and Functional Significance of Runx2 in Hepatocellular Carcinoma: Its Role in Vasculogenic Mimicry and Epithelial- 
Mesenchymal Transition. International journal of molecular sciences 2017, 18(3).

27. Liu C, Liu L, Chen X, Cheng J, Zhang H, Shen J, Shan J, Xu Y, Yang Z, Lai M et al: Sox9 regulates selfrenewal and tumorigenicity by promoting symmetrical cell division of cancer stem cells in hepatocellular carcinoma. Hepatology (Baltimore, Md) 2016, 64(1):117-129.

28. Huang B, Huang M, Li Q: MiR-137 suppresses migration and invasion by targeting EZH2-STAT3 signaling in human hepatocellular carcinoma. Pathology, research and practice 2018, 214(12):19801986.

29. Guo Y, Ma J, Wu L, Wang Q, Li X, Li X, Zhang Y, Zhang J, Yao L, Zhang J et al: Hyperthermia-induced NDRG2 upregulation inhibits the invasion of human hepatocellular carcinoma via suppressing ERK1/2 signaling pathway. PloS one 2013, 8(4):e61079.

30. Luk JM, Burchard J, Zhang C, Liu AM, Wong KF, Shek FH, Lee NP, Fan ST, Poon RT, Ivanovska I et al: DLK1-DIO3 genomic imprinted microRNA cluster at 14q32.2 defines a stemlike subtype of hepatocellular carcinoma associated with poor survival. The Journal of biological chemistry 2011, 286(35):30706-30713.

31. Huan L, Bao C, Chen D, Li Y, Lian J, Ding J, Huang S, Liang L, He X: MicroRNA-127-5p targets the biliverdin reductase B/nuclear factor-kappaB pathway to suppress cell growth in hepatocellular carcinoma cells. Cancer Sci 2016, 107(3):258-266.

32. Xia K, Zhang Y, Cao S, Wu Y, Guo W, Yuan W, Zhang S: miR-411 regulated ITCH expression and promoted cell proliferation in human hepatocellular carcinoma cells. Biomedicine \& pharmacotherapy = Biomedecine \& pharmacotherapie 2015, 70:158-163.

33. Cui W, Huang Z, He H, Gu N, Qin G, Lv J, Zheng T, Sugimoto K, Wu Q: MiR-1188 at the imprinted Dlk1Dio3 domain acts as a tumor suppressor in hepatoma cells. Molecular biology of the cel/ 2015 , 26(8):1416-1427.

34. Yin C, Wang PQ, Xu WP, Yang Y, Zhang Q, Ning BF, Zhang PP, Zhou WP, Xie WF, Chen WS et al: Hepatocyte nuclear factor-4alpha reverses malignancy of hepatocellular carcinoma through regulating miR-134 in the DLK1-DIO3 region. Hepatology (Baltimore, Md) 2013, 58(6):1964-1976.

35. Xu WP, Yi M, Li QQ, Zhou WP, Cong WM, Yang Y, Ning BF, Yin C, Huang ZW, Wang J et al: Perturbation of MicroRNA-370/Lin-28 homolog A/nuclear factor kappa $B$ regulatory circuit contributes to the development of hepatocellular carcinoma. Hepatology (Baltimore, Md) 2013, 58(6):1977-1991.

36. Shimizu M, Sakai H, Shirakami Y, Iwasa J, Yasuda Y, Kubota M, Takai K, Tsurumi H, Tanaka T, Moriwaki H: Acyclic retinoid inhibits diethylnitrosamine-induced liver tumorigenesis in obese and diabetic C57BLKS/J-+(db)/+Lepr(db) mice. Cancer prevention research (Philadelphia, Pa) 2011, 4(1):128-136.

37. Kagawa M, Sano T, Ishibashi N, Hashimoto M, Okuno M, Moriwaki H, Suzuki R, Kohno H, Tanaka T: An acyclic retinoid, NIK-333, inhibits N-diethylnitrosamine-induced rat hepatocarcinogenesis through suppression of TGF-alpha expression and cell proliferation. Carcinogenesis 2004, 25(6):979-985.

38. Funaki M, Kitabayashi J, Shimakami T, Nagata N, Sakai Y, Takegoshi K, Okada H, Murai K, Shirasaki T, Oyama $T$ et al: Peretinoin, an acyclic retinoid, inhibits hepatocarcinogenesis by suppressing 
sphingosine kinase 1 expression in vitro and in vivo. Scientific reports 2017, 7(1):16978.

39. Muto $\mathrm{Y}$, Moriwaki $\mathrm{H}$, Saito A: Prevention of second primary tumors by an acyclic retinoid in patients with hepatocellular carcinoma. The New England journal of medicine 1999, 340(13):1046-1047.

40. Okita K, Izumi N, Matsui O, Tanaka K, Kaneko S, Moriwaki H, Ikeda K, Osaki Y, Numata K, Nakachi K et al: Peretinoin after curative therapy of hepatitis C-related hepatocellular carcinoma: a randomized double-blind placebo-controlled study. Journal of gastroenterology 2015, 50(2):191-202.

41. Liao M, Zhao J, Wang T, Duan J, Zhang Y, Deng X: Role of bile salt in regulating Mcl-1 phosphorylation and chemoresistance in hepatocellular carcinoma cells. Molecular cancer 2011, 10:44.

42. Woolbright $\mathrm{BL}$, Jaeschke $\mathrm{H}$ : Novel insight into mechanisms of cholestatic liver injury. World journal of gastroenterology 2012, 18(36):4985-4993.

43. Kawata S, Takaishi K, Nagase T, Ito N, Matsuda Y, Tamura S, Matsuzawa Y, Tarui S: Increase in the active form of 3-hydroxy-3-methylglutaryl coenzyme A reductase in human hepatocellular carcinoma: possible mechanism for alteration of cholesterol biosynthesis. Cancer research 1990, 50(11):3270-3273.

44. Llovet JM, Burroughs A, Bruix J: Hepatocellular carcinoma. Lancet (London, England) 2003, 362(9399):1907-1917.

45. Morioka S, Sai K, Omori E, Ikeda Y, Matsumoto K, Ninomiya-Tsuji J: TAK1 regulates hepatic lipid homeostasis through SREBP. Oncogene 2016, 35(29):3829-3838.

46. Montero J, Morales A, Llacuna L, Lluis JM, Terrones O, Basanez G, Antonsson B, Prieto J, Garcia-Ruiz C, Colell A et al: Mitochondrial cholesterol contributes to chemotherapy resistance in hepatocellular carcinoma. Cancer research 2008, 68(13):5246-5256.

47. Yeh SH, Chen PJ: Gender disparity of hepatocellular carcinoma: the roles of sex hormones. Oncology 2010, 78 Suppl 1:172-179.

48. Langmead B, Trapnell C, Pop M, Salzberg SL: Ultrafast and memory-efficient alignment of short DNA sequences to the human genome. Genome biology 2009, 10(3):R25.

49. Kozomara A, Griffiths-Jones S: miRBase: annotating high confidence microRNAs using deep sequencing data. Nucleic acids research 2014, 42(Database issue):D68-73.

50. Robinson MD, Oshlack A: A scaling normalization method for differential expression analysis of RNA-seq data. Genome biology 2010, 11(3):R25.

51. Mortazavi A, Williams BA, McCue K, Schaeffer L, Wold B: Mapping and quantifying mammalian transcriptomes by RNA-Seq. Nature methods 2008, 5(7):621-628.

52. Robinson MD, McCarthy DJ, Smyth GK: edgeR: a Bioconductor package for differential expression analysis of digital gene expression data. Bioinformatics (Oxford, England) 2010, 26(1):139-140.

53. Mu Y, Zhou H, Li W, Hu L, Zhang Y: Evaluation of RNA quality in fixed and unembedded mouse embryos by different methods. Experimental and molecular pathology 2013, 95(2):206-212. 
54. Agarwal V, Bell GW, Nam JW, Bartel DP: Predicting effective microRNA target sites in mammalian mRNAs. eLife 2015, 4.

55. Wong N, Wang $X$ : miRDB: an online resource for microRNA target prediction and functional annotations. Nucleic acids research 2015, 43(Database issue):D146-152.

56. Dubchak I, Munoz M, Poliakov A, Salomonis N, Minovitsky S, Bodmer R, Zambon AC: Whole-Genome rVISTA: a tool to determine enrichment of transcription factor binding sites in gene promoters from transcriptomic data. Bioinformatics (Oxford, England) 2013, 29(16):2059-2061.

57. Tang Z, Li C, Kang B, Gao G, Li C, Zhang Z: GEPIA: a web server for cancer and normal gene expression profiling and interactive analyses. Nucleic acids research 2017, 45(W1):W98-W102.

58. Zhou Y, Zhou B, Pache L, Chang M, Khodabakhshi AH, Tanaseichuk O, Benner C, Chanda SK: Metascape provides a biologist-oriented resource for the analysis of systems-level datasets. Nature communications 2019, 10(1):1523.

\section{Tables}

Table 1. Top 20 clusters with their representative enriched terms of target genes of miRNA in DLK1-DIO3 DIR. 


\begin{tabular}{|c|c|c|c|c|c|c|}
\hline Term & Category & Description & Count] & $\% \square$ & $\log 10(P) \square$ & $\log 10(q)$ \\
\hline R-MMU- & Reactome Gene & & & & & \\
\hline 211859 & Sets & Biological oxidations & 33 & 12.22 & -22.43 & -18.17 \\
\hline \multirow{3}{*}{ GO:0032787 } & GO Biological & \multirow[b]{2}{*}{ monocarboxylic acid metabolic process } & \multirow[b]{2}{*}{39} & \multirow[b]{2}{*}{14.44} & \multirow[b]{2}{*}{-16.67} & \multirow[b]{2}{*}{-12.72} \\
\hline & Processes & & & & & \\
\hline & GO Biological & \multirow[b]{2}{*}{ drug metabolic process } & \multirow[b]{2}{*}{42} & \multirow[b]{2}{*}{15.56} & \multirow[b]{2}{*}{-15.33} & \multirow[b]{2}{*}{-11.67} \\
\hline GO:0017144 & Processes & & & & & \\
\hline \multirow{2}{*}{$\begin{array}{l}\text { R-MMU- } \\
71291\end{array}$} & Reactome & \multirow{2}{*}{ Metabolism of amino acids and derivatives } & \multirow[b]{2}{*}{23} & \multirow[b]{2}{*}{8.52} & \multirow[b]{2}{*}{-14.18} & \multirow[b]{2}{*}{-10.62} \\
\hline & Sets & & & & & \\
\hline \multirow[t]{2}{*}{ mmu00830 } & KEGG Pathway & Retinol metabolism & 15 & 5.56 & -12.1 & -8.68 \\
\hline & GO Biological & \multirow[b]{2}{*}{ cofactor metabolic process } & \multirow[b]{2}{*}{30} & & & \\
\hline GO:0051186 & Processes & & & 11.11 & -11.43 & -8.13 \\
\hline R-MMU- & Reactome Gene & & & & & \\
\hline 194068 & Sets & Bile acid and bile salt metabolism & 11 & 4.07 & -10.24 & -7.02 \\
\hline & GO Biological & & & & & \\
\hline GO:0010817 & Processes & regulation of hormone levels & 27 & 10 & -8.22 & -5.34 \\
\hline mmu00983 & KEGG Pathway & Drug metabolism - other enzymes & 9 & 3.33 & -7.66 & -4.86 \\
\hline & GO Biological & & & & & \\
\hline GO:0008202 & Processes & steroid metabolic process & 18 & 6.67 & -7.1 & -4.41 \\
\hline $\begin{array}{l}\text { R-MMU- } \\
6788656\end{array}$ & $\begin{array}{l}\text { Reactome Gene } \\
\text { Sets }\end{array}$ & $\begin{array}{l}\text { Histidine, lysine, phenylalanine, tyrosine, proline and } \\
\text { tryptophan catabolism }\end{array}$ & 8 & 2.96 & -6.98 & -4.31 \\
\hline R-MMU- & Reactome Gene & & & & & \\
\hline 1614635 & Sets & Sulfur amino acid metabolism & 6 & 2.22 & -6.48 & -3.87 \\
\hline GO:0016042 & $\begin{array}{l}\text { GO Biological } \\
\text { Processes }\end{array}$ & lipid catabolic process & 16 & 5.93 & -5.49 & -3.03 \\
\hline & GO Biological & & & & & \\
\hline GO:0006536 & Processes & glutamate metabolic process & 6 & 2.22 & -5.44 & -3 \\
\hline & GO Biological & & & & & \\
\hline GO:0060749 & Processes & mammary gland alveolus development & 5 & 1.85 & -5.05 & -2.66 \\
\hline R-MMU- & Reactome & & & & & \\
\hline 192105 & Sets & Synthesis of bile acids and bile salts & 6 & 2.22 & -4.99 & -2.62 \\
\hline & GO Biological & & & & & \\
\hline GO:0001505 & Processes & regulation of neurotransmitter levels & 17 & 6.3 & -4.99 & -2.62 \\
\hline & GO Biological & & & & & \\
\hline GO:0060736 & Processes & prostate gland growth & 4 & 1.48 & -4.56 & -2.24 \\
\hline & GO Biological & & & & & \\
\hline GO:0050433 & Processes & regulation of catecholamine secretion & 7 & 2.59 & -4.35 & -2.07 \\
\hline & GO Biological & & & & & \\
\hline GO:1901361 & Processes & organic cyclic compound catabolic process & 19 & 7.04 & -4.34 & -2.07 \\
\hline
\end{tabular}

I. "Count" is the number of genes in the user-provided lists with membership in the given ontology term.

e percentage of all of the user-provided genes that are found in the given ontology term (only input genes with at least one ontology term annotation led in the calculation).

प" $\log 10(\mathrm{P})$ " is the p-value in $\log$ base 10.

प"Log10(q)" is the multi-test adjusted p-value in log base.

\section{Supplemental Table And Figure Legends}

\section{Supplemental Tables}

Table S1. Raw and clean reads obtained by NGS.

Table S2. DEMIRs in males (191) and females (204). 
Table S3. Common and unique DEMIRs in different expression patterns.

Table S4. Up-regulated and down-regulated mRNAs selected for the 3-node FFL co-regulatory network analysis.

Table S5. Relationships between HCC-related genes, miRNAs, and TFs.

Table S6. Relationships between TFs, miRNAs, and genes.

Table S7. Summary of 3-node FFL based on CRC-related prediction data.

Table S8. HCC-related genes in miRNA-FFLs, TF-FFLs, and composite-FFLs.

Table S9. DEMIRs in the DIk1-Dio3 DIR.

Table S10. Metascape analysis for down-regulated mRNAs corresponding to up-regulated miRNAs in the DIk1-Dio3 DIR of both males and females.

Table S11. The miRNA specific 5'-primer sequences of selected DEMIRs and internal control.

\section{Supplemental Figure legends}

Fig. S1. Experimental flowchart. The microRNA and mRNA expression profiles of tissue samples from male and female mice were determined by next-generation sequencing. Following vertical and horizontal comparison, expression profiling, 3-node-FFL, DLK1-DIO3 GIR, and pathway enrichment analysis were performed. MW and FW, wild-type liver tissues of males and females, respectively; MP and FP, peri-tumor tissues of males and females, respectively; MT and FT, hepatic tumor tissues of males and females, respectively; "/", comparison between former and latter; DEMIRs, differentially expressed microRNAs; DEMRs, differentially expressed mRNAs; GIR, genomic imprinting region; FFL, feed-forward loop.

Fig. S2. Saturation analysis for detected miRNAs mapped by clean reads.

Fig. S3. The expression pattern analysis of total DEMIRs for hepatocarcinogenesis. The description is same as Fig. 4E. Specially, the white column in expression models indicates that the miRNA levels in this tissue have no significant difference compared with other tissues.

\section{Figures}


A

\begin{tabular}{|c|c|c|c|c|c|c|}
\hline $\begin{array}{c}\text { Pearon's } \\
\text { correlation }\end{array}$ & MW & MP & MT & FW & FP & FT \\
\hline MW & 1.00 & 0.89 & 0.88 & 0.87 & 0.89 & 0.86 \\
\hline MP & - & 1.00 & 0.87 & 0.88 & 0.89 & 0.86 \\
\hline MT & - & - & 1.00 & 0.85 & 0.87 & 0.89 \\
\hline FW & - & - & - & 1.00 & 0.88 & 0.82 \\
\hline FP & - & - & - & - & 1.00 & 0.85 \\
\hline FT & - & - & - & - & - & 1.00 \\
\hline
\end{tabular}

B

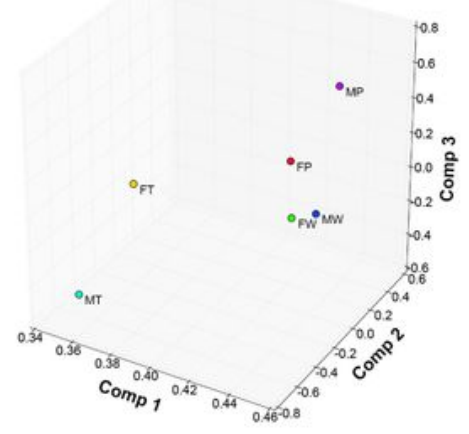

C

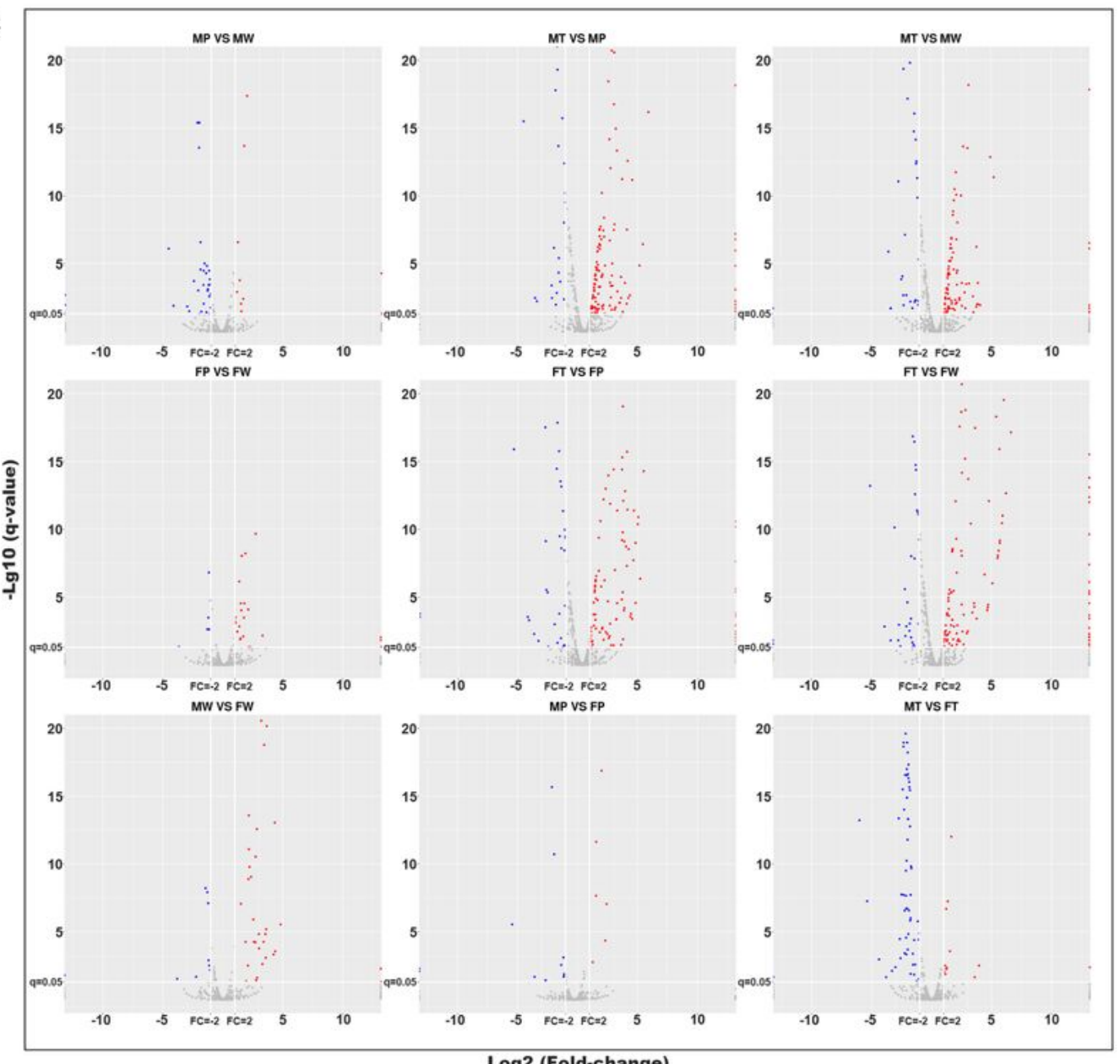

Log2 (Fold-change)

\section{Figure 1}

The Pearson's correlation, PCA and DEMIRs analysis for microRNA omics data. (A) Heatmap of the Pearson's correlation (R2) analysis of microRNA omics dataset. Values denote the Pearson correlation coefficients. (B) Principal component analysis (PCA) calculation results are expressed as 3D scatter plot. (C) Volcano plot of pairwise comparisons. Red and blue colors represent relatively up- and downregulated miRNAs, respectively. MW and FW, wild-type liver tissues of males and females, respectively; 
MP and FP, peri-tumor tissues of males and females, respectively; MT and FT, hepatic tumor tissues of males and females, respectively.
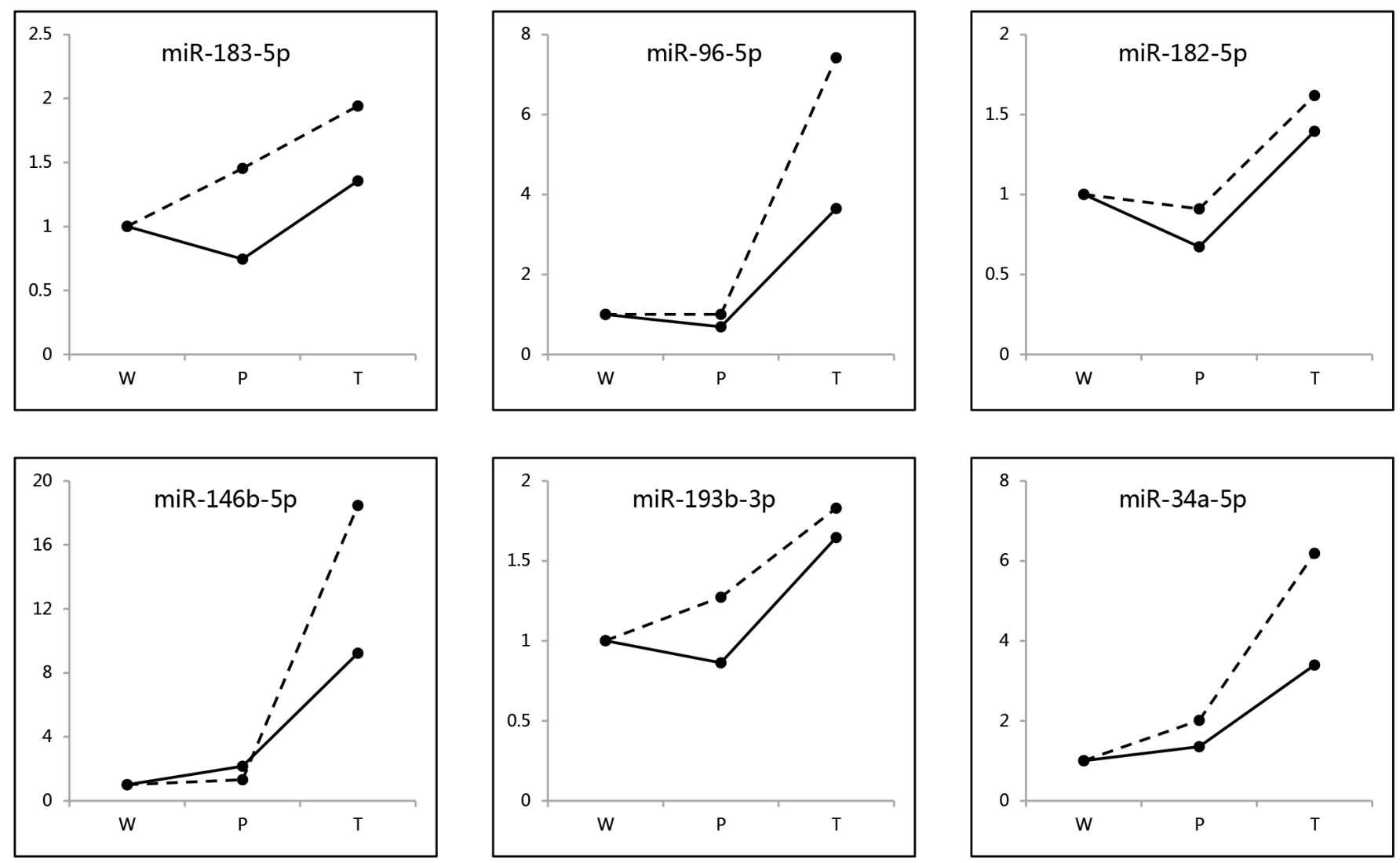

\section{Figure 2}

Validation for DEMIRs by RT-qPCR. Six randomly selected DEMIRs detected in both sexes were evaluated by RT-qPCR analysis. The primer sequences were presented in Table S11. Hidden line, males; Solid line, females. 
A

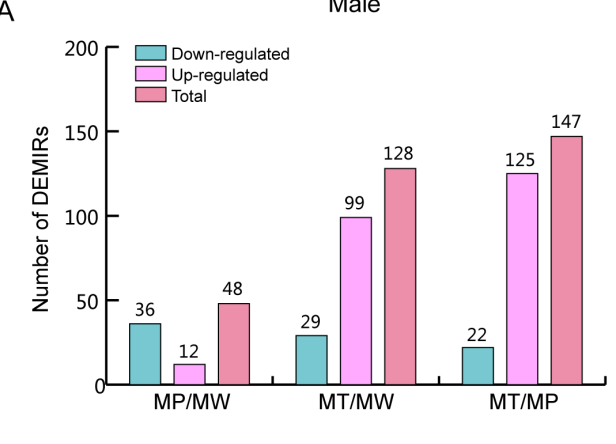

C

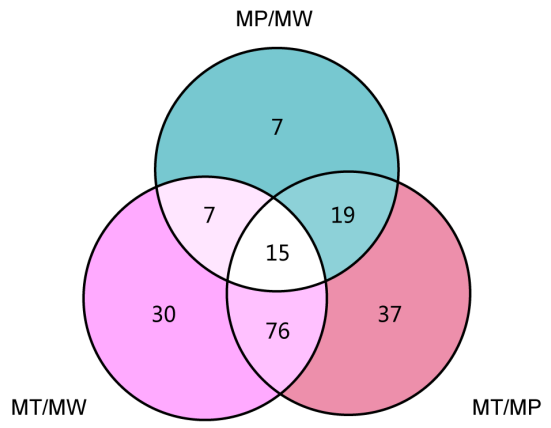

B

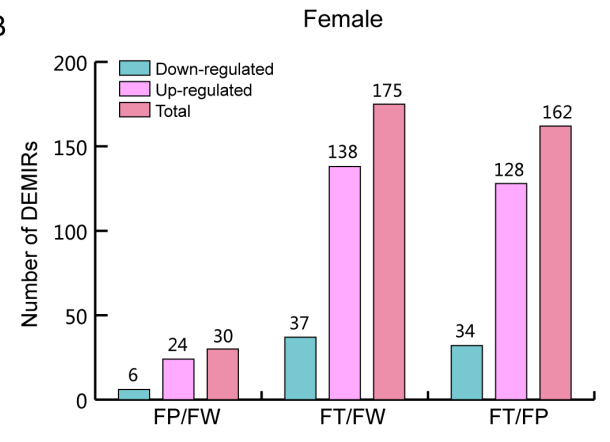

D

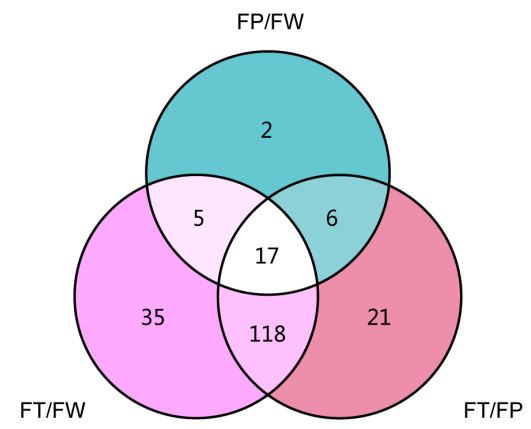

E

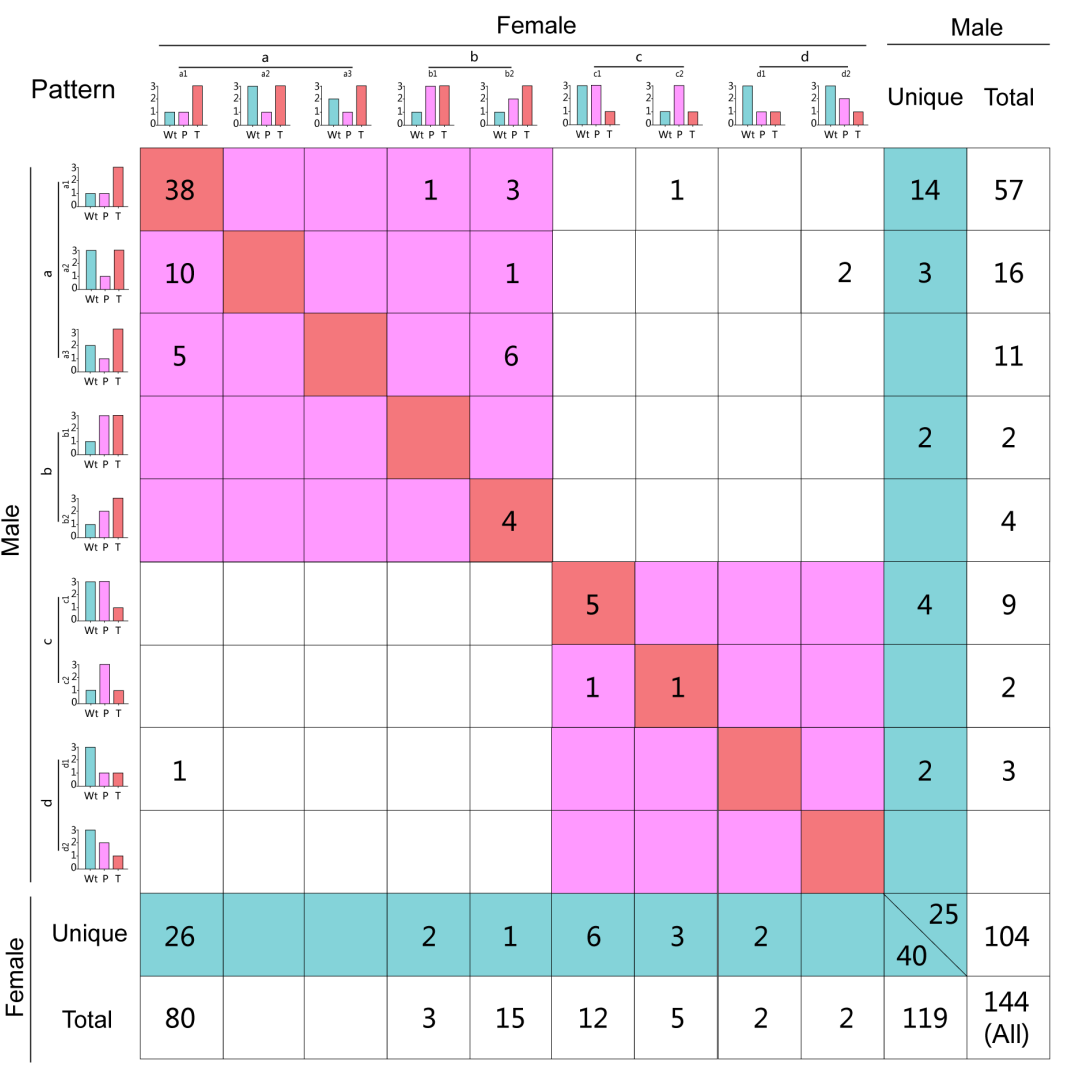

\section{Figure 3}

Differentially expressed miRNAs (DEMIRs) obtained by vertical comparison and expression pattern analysis for hepatocarcinogenesis. (A) The number of miRNAs that are expressed at higher or lower levels when comparing MP/MW, MT/MW, and MT/MP in male mice. (B) The number of up- and downregulated miRNAs when comparing FP/FW, FT/FW, and FT/FP in female mice. (C) Venn analysis of the DEMIRs shown in Fig. 4A. (D) Venn analysis of the DEMIRs shown in Fig. 4B. (E) Expression pattern 
analysis for hepatocarcinogenesis for both sexes. The diagonal line indicates that these miRNAs have the same expression patterns in both sexes. The miRNAs shown in the red area in the upper left are upregulated in $\mathrm{T}$ as compared to $\mathrm{P}$ and/or $\mathrm{W}$ in both sexes. The miRNAs shown in the red area in the bottom right are down-regulated in $T$ as compared to $P$ and/or $W$ in both sexes. The miRNAs shown in the blue area have a unique expression pattern in males and females. The relative expression levels of miRNAs are represented using a 1,2, and 3 grading system, indicating significant differences in the miRNA levels. The DEMIRs that appeared at least two times in pairwise comparison among $\mathrm{W}, \mathrm{P}$, and $\mathrm{T}$ between sexes are shown here, and the expression pattern analysis for total DEMIRs is shown in Fig. S3. 
I Compilation of $\mathrm{HCC}$ related genes and miRNAs

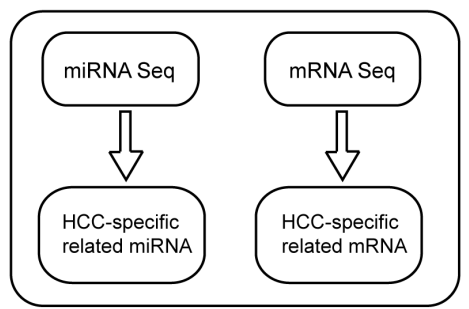

IV Construction of the common regulatory networks
II Prediction of regulatory relationships among TFs, miRNAs and genes

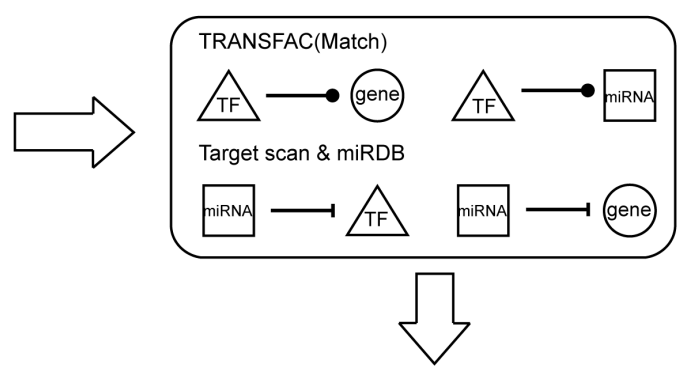

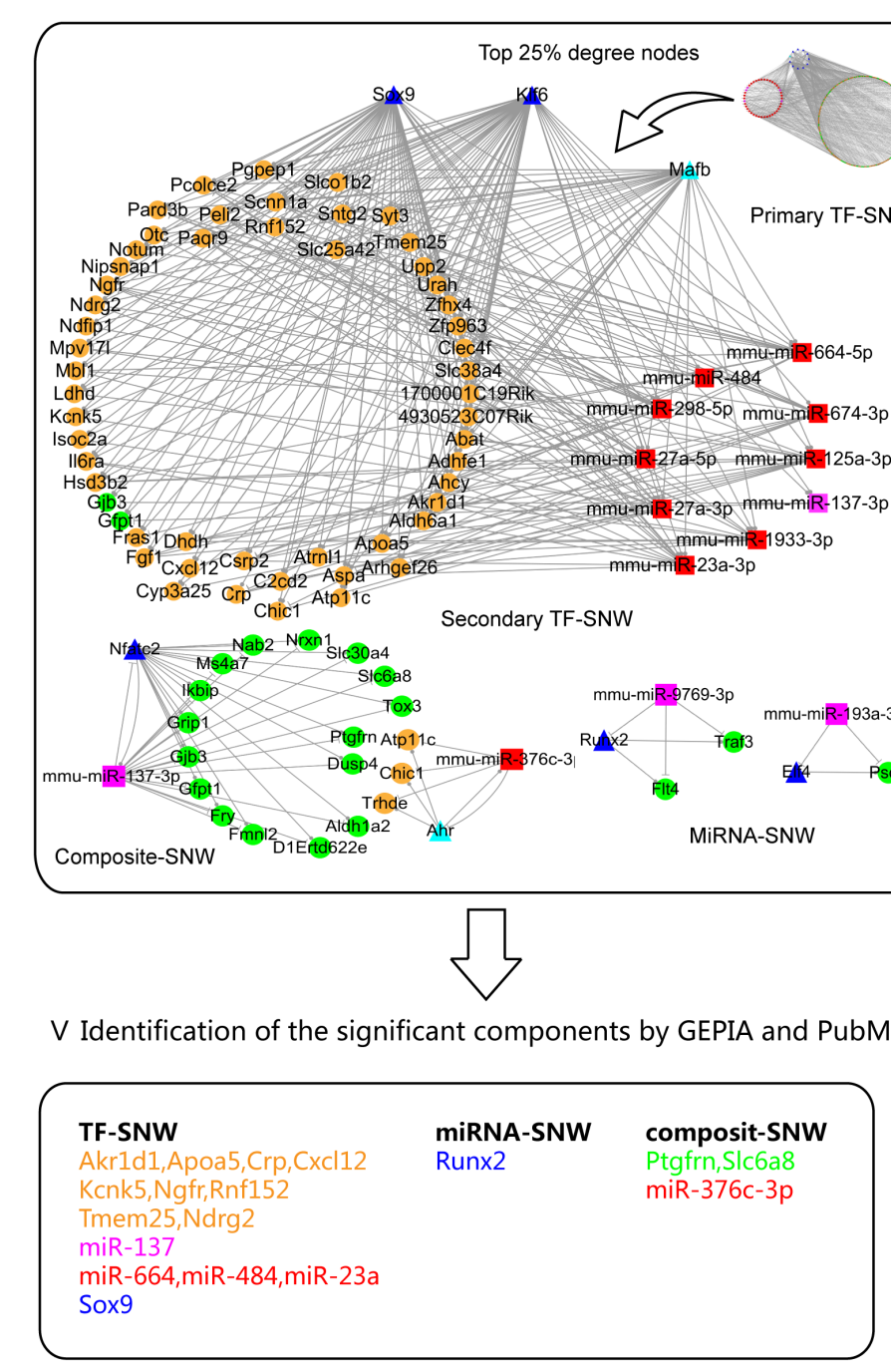

III Identification of significant FFLs
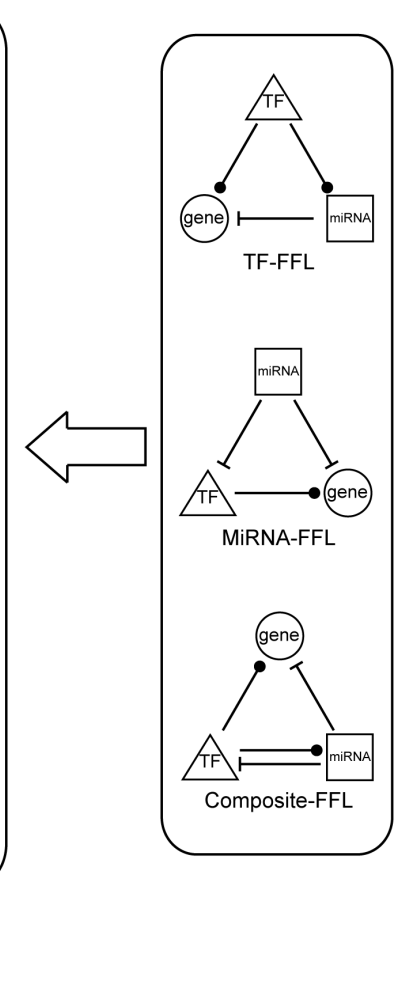
genes. The four regulatory relationships include TF-gene, TF-miRNA, miRNA-gene, miRNA-TF. (III) Identification of significant FFLs. Based on the above regulatory relationships, the significant 3-node FFLs were identified. (IV) Construction of the shared regulatory networks. These FFLs were categorized into TF, miRNA, and composite sub-networks (SNWs). Among these three SNWs, TF-SNW is the most redundant. By using the hub definition method, we determined the top $25 \%$ degree nodes of genes, miRNAs, and TFs as the member of secondary TF-SNW to focus on the most significant components. (V) Identification of the significant components using GEPIA and PubMed database. All nodes in the three SNWs (secondary TF-SNW, miRNA-SNW, composite-SNW) were filtered based on the GEPIA and PubMed database. 
A

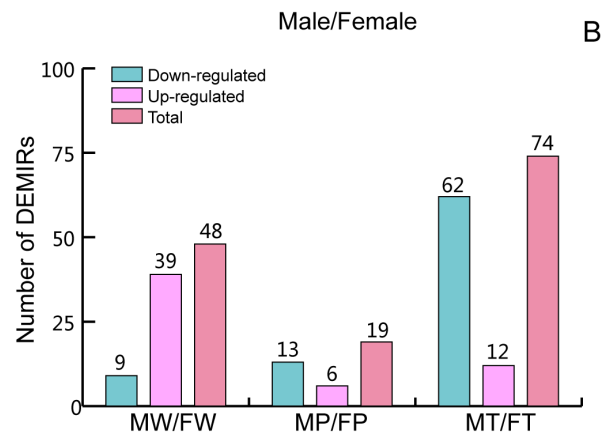

B

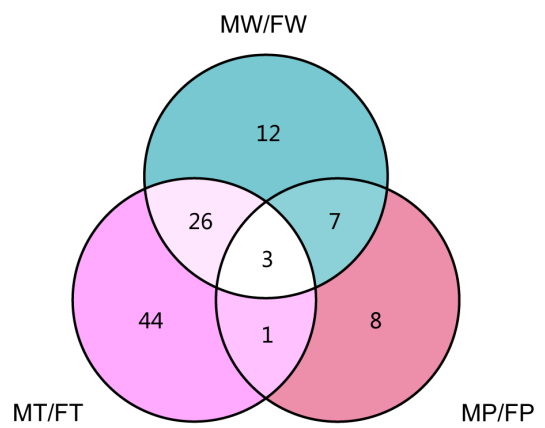

C
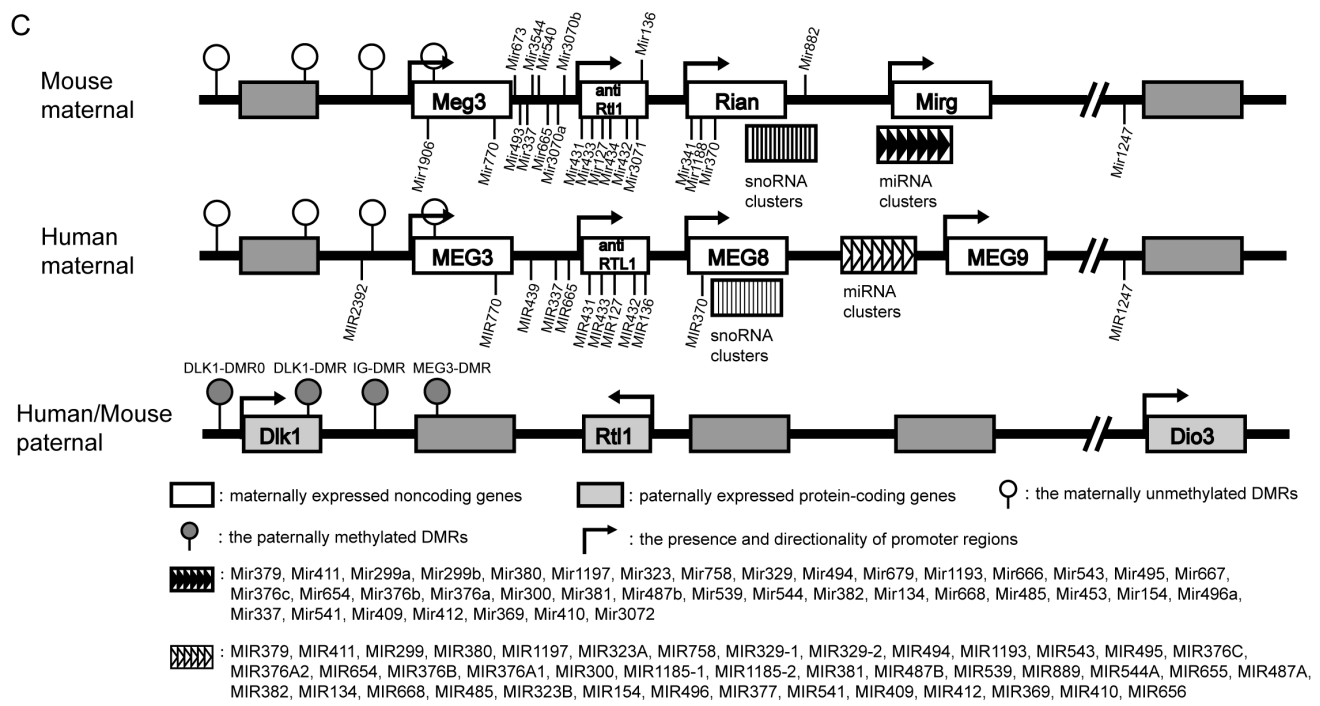

$\mathrm{D}$

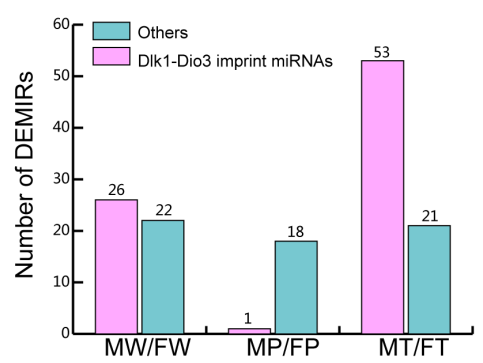

F

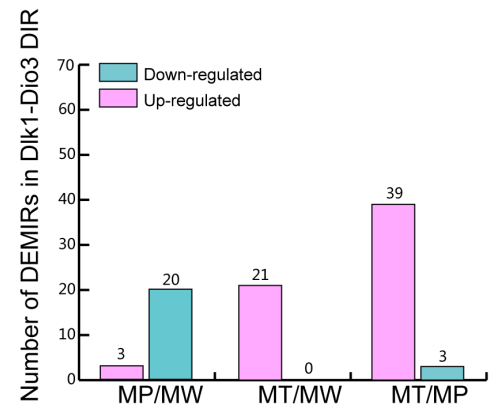

E

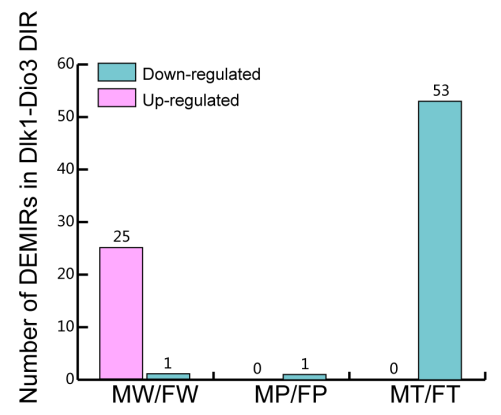

G

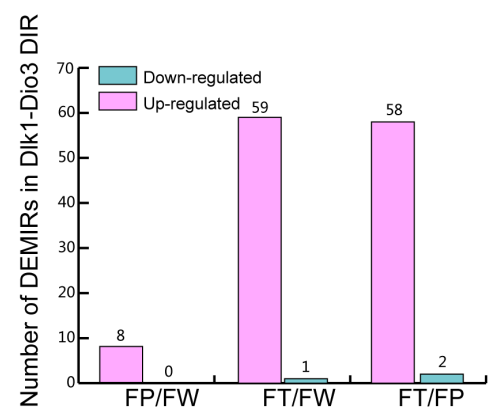

\section{Figure 5}

DEMIRs between sexes in the process of hepatocarcinogenesis. (A) The number of up- and downregulated miRNAs in the comparisons in MW/FW, MP/FP, and MT/FT between sexes. (B) Venn analysis of DEMIRs in (A). (C) Comparative structure model of Dlk1-Dio3 genomic imprinting region (GIR) between human and mice. (D) The number of DEMIRs located in and beyond DIk1-Dio3 GIR. (E) The number of upand down-regulated miRNAs located on DIk1-Dio3 GIR between sexes. (F) The number of up- and down- 
regulated miRNAs located on DIk1-Dio3 GIR in males. (G) The number of up- and down-regulated miRNAs located on DIk1-Dio3 GIR in females.

\section{Supplementary Files}

This is a list of supplementary files associated with this preprint. Click to download.

- TableS8.xisx

- TableS2.xIsx

- TableS3.xIsx

- TableS5.xIsx

- TableS6.xlsx

- TableS4.xlsx

- TableS11.xlsx

- theARRIVEguidelineschecklist20200204.pdf

- Tables10.xlsx

- Fig.S3.tif

- Fig.S2.tif

- TableS1.xlsx

- Fig.S1.tif

- TableS9.xIsx

- TableS7.xlsx 